Journal of Multidisciplinary Dental Research

Case Report

\title{
Ridge augmentation of Class III ridge defect using piezosurgery with autogenous chin graft followed by implant placement: A 6 months follow-up interventional study
}

\author{
Namburi Rajesh ${ }^{1}$, Rajendran Poornima ${ }^{2, *}$ \\ ${ }^{1}$ Assistant Professor, Department of Dental Sciences, Maharaja Institute of Medical Sciences, Nellimarla, Vizianagaram, Andhra \\ Pradesh, India \\ ${ }^{2}$ Senior Lecturer, Department of Periodontology, Sri Ramakrishna Dental College and Hospital, Coimbatore, Tamil Nadu, India
}

\section{A RTICLE INFO}

Article history:

Received 19.12.2020

Accepted 18.01.2021

Published 19.01.2021

\section{${ }^{*}$ Corresponding author.}

Rajendran Poornima

poorni89.27@gmail.com

https://doi.org/

$10.38138 / \mathrm{JMDR} / \mathrm{v} 6 \mathrm{i} 2.9$

\begin{abstract}
A B S T R A C T
Background: Tooth loss occurs due to periodontal disease, extensive dental caries, and fracture of teeth after a dento-alveolar trauma, cyst and tumours of odontogenic and non-odontogenic origin. Following loss of teeth, alveolar ridge resorption becomes an unwanted but unavoidable consequence. In order to restore the lost bone and replace the teeth with implants, ridge augmentation procedures are done. The present study aimed at restoring the class III ridge defect using piezosurgery with autogenous block bone graft procured from mandibular symphysis region. Materials and methods: A total number of 10 healthy patients with Class III ridge defect in maxillary anterior teeth region were enrolled for the study. The donor and defect sites were pre-surgically analysed using Cone-Beam Computed Tomography (CBCT), preclinical models and ridge mapping. The implant site width and height were assessed intra-operatively during the surgery and at re-entry after 6 months. The autogenous block bone grafts were procured from mandibular chin region using piezo-electric device and placed at the recipient site. At the 6 months re-evaluation, both clinical and radiographic parameters were assessed using CBCT of the recipient site. Implants were placed at two-stages and followed-up up to 6 months. Results: Clinical measurements showed that the mean horizontal bone gain and vertical bone gain was noted to be $3.21 \pm 0.24 \mathrm{~mm}$ and $2.18 \pm 0.32 \mathrm{~mm}$ respectively, by the end of 6 months follow-up. No major complications were presented at the recipient as well as donor sites. Piezoelectric surgery provided clean, bloodless field during the procedure and minimal post-operative discomfort to the patients. Conclusion: The results of the present study suggests that, class III ridge defects can be successfully augmented using piezo-surgery using autogenous bone grafting from the mandibular symphysis region. Also, delayed implant placement may provide satisfactory functional and aesthetic outcomes on the long term.
\end{abstract}

Keywords: Ridge augmentation; chin graft; Piezosurgery; dental implants

\section{INTRODUCTION}

Dental implant therapy has become the current and future trend for replacement of missing teeth. However, the biggest challenge faced by implant dentistry is excessive alveolar ridge resorption following extraction of teeth. In dental clinical practice, deficiency of bone height/width, volume is one of the prime reasons for evading implant therapy.

In order to make the alveolar ridge adequate for implant placement, it is essential to re-establish the ridge dimension favourably. This could be achieved using bone-grafting materials. Augmenting the alveolar ridge is the process of appending bone in anatomically and/or functionally deficient regions. The purpose of the grafted material is to provide stability and support for the future dental implant. As the innate bone grows, it substitutes the grafted material and eventually results in an amalgamated region of new bone. ${ }^{(1)}$

Regardless of the recent advances in bone grafts and bone-substitutes, intramembranous autogenous bone grafts harvested from intra-oral or extra-oral sites are deemed as the gold-standards for alveolar ridge reconstruction. ${ }^{(2)}$ If the quantity of bone needed for augmentation is moderate, grafts can be procured from intra-oral regional sites such 
as maxillary tuberosity, mandibular symphysis, mandibular ramus, angle of mandible and any exostoses.

Block grafts obtained from mandibular symphysis can be used predictably for ridge augmentation up to $6 \mathrm{~mm}$ in horizontal and vertical dimension. ${ }^{(1)}$ The bone quality usually found in this region is D-1 or D-2 and grafts for augmenting up to three teeth edentulous areas can be obtained from the chin region. Pikos M in 2000 conducted a study assessing the efficiency of block grafts in restoring the ridge defects. Amongst 115 autogenous block grafting, only one complete failure was noted and other cases showed predictable clinical outcome. ${ }^{(3)}$

Furthermore, the use of piezosurgery in periodontal and implant therapy simplifies and improves handling of soft and hard tissues. However, the introduction of piezosurgery has enabled the manual instrumentation limits to be pushed, and this makes the procedure a simpler and more reliable technique. The key benefits of the piezoelectric instrument are precise cut on hard tissues and its ability to cause minimal tissue damage ensuing better healing. ${ }^{(4,5)}$

Thus, the aim of the present study is to evaluate the amount of horizontal and vertical bone gain achieved by ridge augmentation procedure with autogenous bone graft procured from mandibular symphysis region using piezosurgery unit.

\section{MATERIALS AND METHODS}

\section{Study sample}

A total number of ten patients who reported with chief complaint of missing upper teeth with Sibert's Class III ridge defect $^{(6)}$ were included in the study (Figure 1). Institutional ethical committee approval was secured before initiating the study. The patients were explained about the methodology of the study and informed consents were obtained. All the patients were assessed for any relevant medical history and 10 systemically healthy patients ( 6 male and 4 female) aged between 20 and 50 years were preferred for the study. The patients with Class I and II ridge defects, oral abusive habits such as smoking, pan chewing, pregnant women and lactating mothers were excluded from the study.

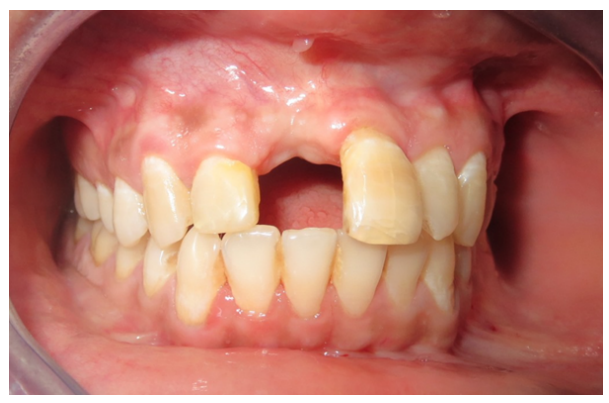

Fig. 1: pre-operative mage of the ridge defect following extraction of the fractured tooth
Pre-operative examination encompassed clinical and radiographic examination of the donor and the implant recipient site. Clinically, ridge mapping was done to assess the existing ridge width and height in the recipient site. This was further confirmed by analysing the pre-clinical models. The baseline horizontal ridge width and vertical ridge height values are $3.21 \pm 0.24 \mathrm{~mm}$ and $6.18 \pm 0.32 \mathrm{~mm}$ respectively. The donor site was examined for ridge morphology, vestibular depth, and width of keratinized tissue and thickness of attached gingiva. Intra-oral periapical radiographs (IOPAR) and Cone-beam Computed Tomography (CBCT) analysis of both the recipient and the donor site was carried out. This enabled in knowing the quantity of bone required for the recipient site and quality as well as quantity of bone available in the donor site.

\section{Surgical procedure}

Following the preparation of the surgical area and setting up the armamentarium, the recipient sites were anesthetized using 2\% lignocaine hydrochloride with 1:100000 adrenaline. The edentulous site requires initial mid-crestal incision that continues into the sulcus as sulcular incision on either side of the defect. Bilateral vertical releasing incisions are given and full thickness mucoperiosteal flap is reflected (Figure 2). The sites were further prepared by performing decortication using a 702L straight fissure bone cutting bur.

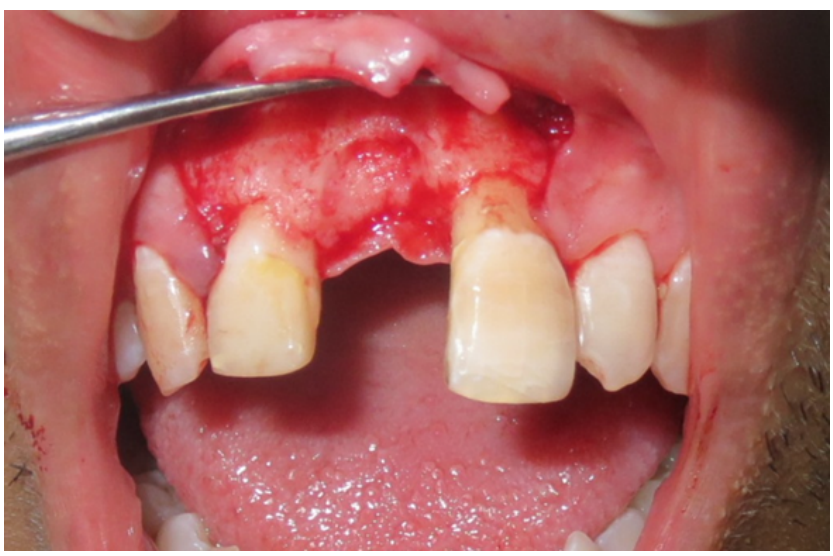

Fig. 2: Preparation of the recipient site after full-thickness mucoperiosteal flap reflection

The donor site was anesthetised by bilateral mandibular block, mental nerve block and infiltration in the midline at the base of the mental protuberance. Mucoperiosteal flap was reflected using sulcular approach. The lateral extent of graft to be harvested was marked using osteotomy 7 piezosurgery tip (OT7) (Figure 3). For block harvesting from the symphysis, guidelines proposed by Pommer et al. in $2008^{(7)}$ to curtail neurosensory injury. After amputating the graft from the surrounding bone, it is stored in saline until it is transplanted into the recipient site. The area is then filled with collagen sponges, particulate alloplastic bone graft and guided tissue regeneration (GTR) membrane (Figure 4).

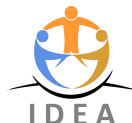


The flap is then re-approximated and sutured with simple interrupted sutures.

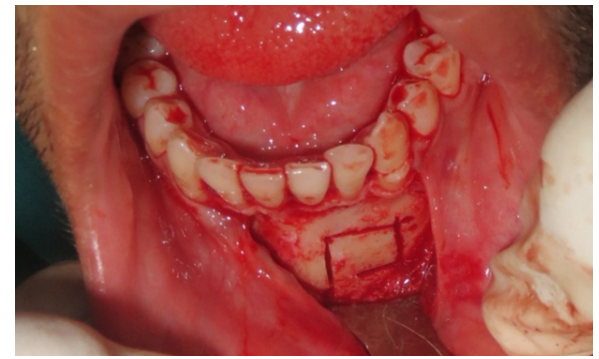

Fig. 3: Donor site preparation using sulcular release approach in the mandibular symphysis region. The outline of desired size of block graft required is marked using piezo-electric device

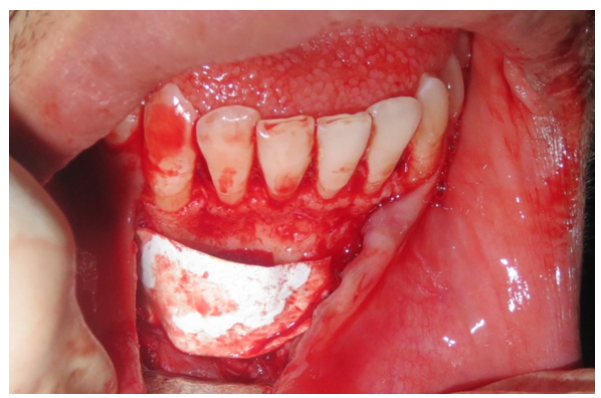

Fig. 4: Donor site packed with collagen sponges, particulate allograft bone substitute and GTR membrane

The autogenous block chin graft is then placed in the recipient site and secured using two titanium screws to prevent micro-rotation of the graft. Autogenous particulate bone graft procured from the donor site was mixed with alloplastic particulate bone graft material and then packed surrounding the block graft (Figure 5). The recipient site was then covered by a GTR membrane and the mucoperiosteal flap was slightly coronally advanced and sutured using 4-0 silk suture material (Figure 6).

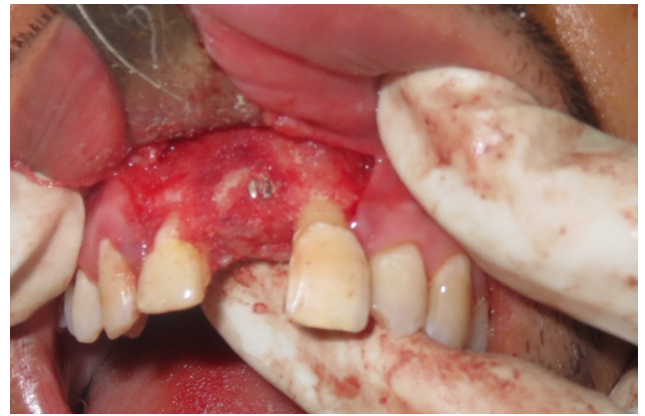

Fig. 5: the procured block graft is placed in the recipient site and secured using titanium screws. Particulate autogenous graft material mixed with alloplast is placed surrounding the block graft

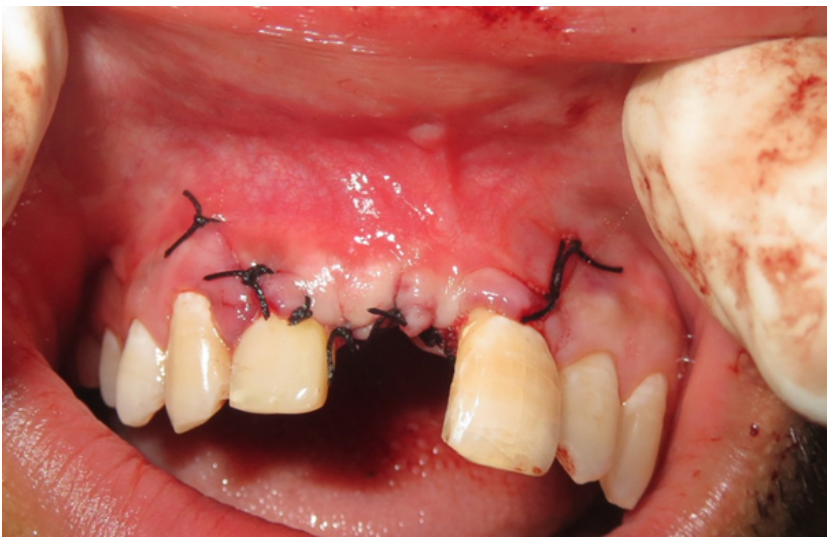

Fig. 6: The recipient site is sutured after flap advancement and approximation using 4-0 silk suture material by simple interrupted sutures

All the patients were given post-operative instructions and were prescribed with analgesics (Ibuprofen $400 \mathrm{mg}$ BID for 3 days), antibiotics (Amoxicillin with clavulanic acid; 500 mg+625 mg TID for 5 days) and chlorhexidine digluconate (0.2\% BID for two weeks) for chemical plaque control. Suture removal was done around 14 days post-operatively. The patients were recalled after 6 months to re-assess the ridges clinically and radiographically for surgical re-entry and implant placement.

At the 6 months follow-up, surgical re-entry was done with flap outline similar to the first surgery. Subsequent to mucoperiosteal flap elevation, the healed crest width and height were measured and recorded. The fixation screws that held the bone blocks were removed, and implant insertion was performed according to standard surgical protocols (Figures 7 and 8). The implants were then restored after a healing period of 4-6 months (Figure 9).

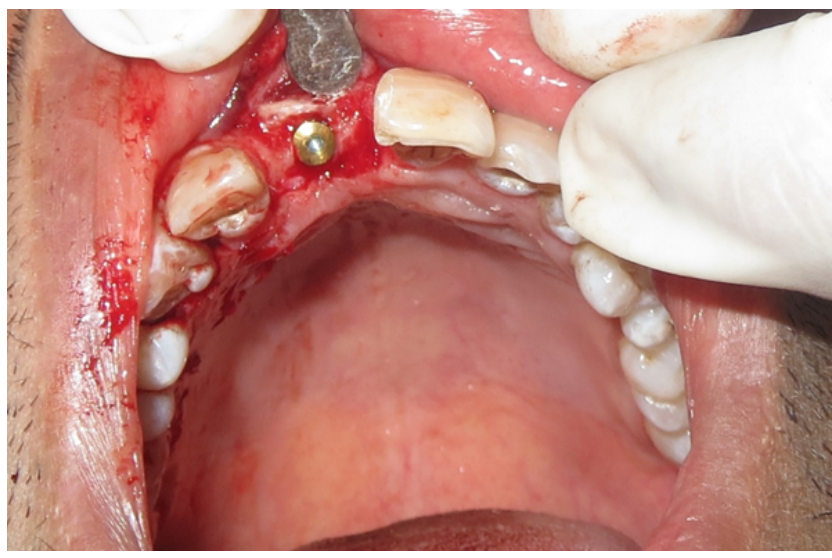

Fig. 7: Implant placed after 6 months on surgical re-entry of the augmented site

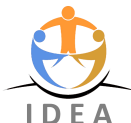




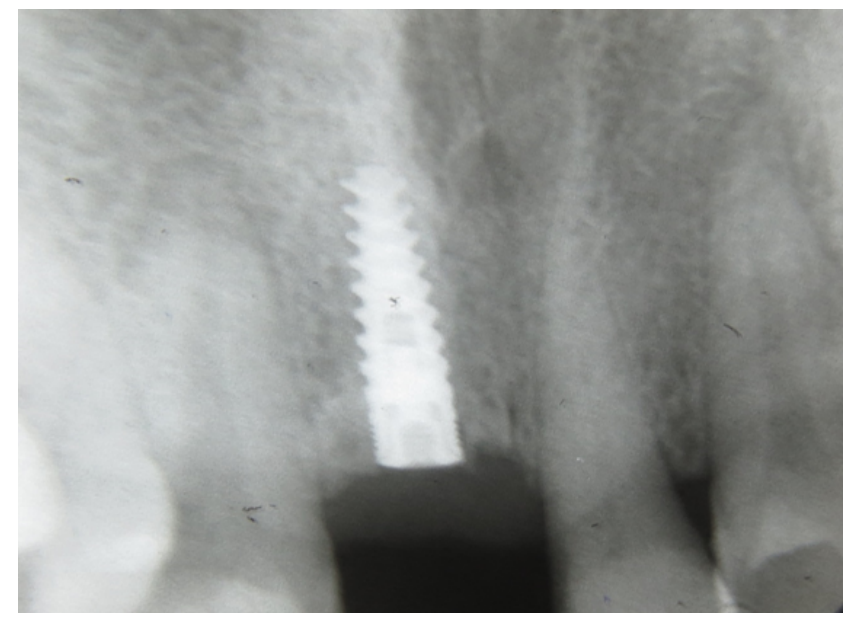

Fig. 8: IOPAR showing the augmented site with complete trabecular pattern formation and implant after placement

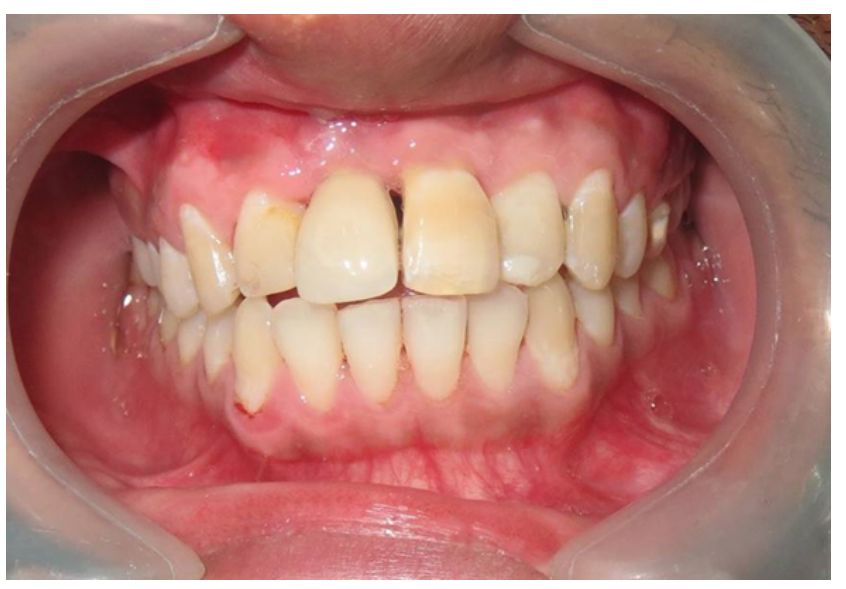

Fig. 9: Implant loaded after 6 months of placement

\section{Statistical analysis}

All the data of the clinical parameters assessed were first probed descriptively and were articulated as median and mean values. Statistical significance for the clinical parameters at baseline and at 6 months follow-up period was determined by the nonparametric ANOVA test using the Brunner-Langer model for longitudinal data. $\mathrm{P}$ value of less than $0.05(\mathrm{P} \leq 0.05)$ was regarded statistically significant. All statistical analyses were computed using SPSS software version 18.

\section{RESULTS}

Out of 10 patients, 8 patients did not report any significant adverse effect at the donor site. 2 patients had postoperative discomforts that included swelling and pain for the first week. However, they did not require specific additional treatment. The recipient site in 9 out of 10 patients showed uneventful healing, whereas one patient developed hematoma for which antibiotic therapy was extended for 2 more days. Clinical parameters recorded at baseline and at 6 months follow-up showed that the gain in horizontal ridge width was $6.11 \pm 0.54 \mathrm{~mm}$ and the gain in vertical ridge height was $9.72 \pm 0.98 \mathrm{~mm}$. The clinical parameters showed statistically significant improvement from baseline to postoperative 6 months with $\mathrm{P}$ value $\leq 0.05$ (Table 1).

Table 1: Showing the clinical parameters that were assessed pre-operatively and at 6 months post-operatively

\begin{tabular}{llll}
\hline Parameter & Values & & \\
\hline $\begin{array}{l}\text { Mean patient age } \\
\text { Male: female }\end{array}$ & 31.5 years & & \\
patient ratio & $6: 4$ & & \\
& Pre- & $\begin{array}{l}\text { Post-operative } \\
\text { operative }\end{array}$ & $\mathrm{P}$ \\
& $(6$ months $)$ & value \\
$\begin{array}{l}\text { Mean horizontal } \\
\text { ridge width }\end{array}$ & $\begin{array}{l}\mathrm{mm} \pm 0.24 \\
\text { Mean vertical }\end{array}$ & $6.11 \pm 0.54 \mathrm{~mm}$ & $\leq 0.05$ \\
ridge width & $\mathrm{mm}$ & $9.72 \pm 0.98 \mathrm{~mm}$ & $\leq 0.05$ \\
\hline
\end{tabular}

The level of statistical significance was set at $\mathrm{P} \leq 0.05$ and statistical analysis was done by repeated measures ANOVA.

\section{DISCUSSION}

Once a natural tooth is lost, there will be inevitable resorption of alveolar ridge that may jeopardise the aesthetics and alter the function of adjacent teeth. When pondering the treatment options for replacement of teeth missing from the anterior region of both maxilla and mandible, the main objective is always to provide a functional restoration that is synchronous with rest of the natural dentition.

When the remaining bone level is not adequate for immediate replacement of the missing teeth, bone augmentation procedures are employed. In order to attain bone regeneration, the principles of osteogenesis, osteoinduction, osteoconduction and osteostimulation can be applied to heighten the therapeutic methodologies. ${ }^{(8)}$

Seibert JS in 1983 gave the ridge defect classification where class I defect has bucco-lingual loss of alveolar bone resulting in reduced ridge width with normal ridge height, class II defect has reduced bone height with apico-coronal loss of bone and normal ridge width, class III defect shows combination of both reduced ridge height and width with loss of bone apico-coronally and bucco-lingually. ${ }^{(6)}$

Class III defects were chosen for the present study as it is challenging to achieve bone regeneration in both horizontal and vertical directions. Autogenous block bone grafts in combination with particulate autogenous grafts, alloplast and GTR has provided promising results. ${ }^{(9)}$ Symphysis region was chosen as the donor site as it is formed by intramembranous bone formation with $\mathrm{D}-1$ or $\mathrm{D}-2$ type of bone and provides easier access to procure the graft than other intra-oral donor sites. ${ }^{(10,11)}$ Moreover,

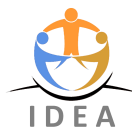


autogenous grafts are the only grafts that aids in new bone formation by promoting osteogenesis and osteoinduction in the recipient site. Therefore, they are regarded as the gold standard, for increasing the bone volume and enable implant placement. ${ }^{(12)}$ Hence, in the present study, autogenous block bone graft from mandibular symphyseal region in combination with particulate autograft, alloplast and GTR membrane was employed.

Chappuis in 2016 evaluated the lateral ridge augmentation using autogenous block grafts and guided bone regeneration (GBR) and followed up the cases for a period 10 years. The results demonstrated that lateral ridge augmentation using block bone grafts and GBR demonstrated a high success rate of $98.1 \%$ with minimal graft resorption noted at 10 years. ${ }^{(9)}$ Proussaefs in 2005 used intra-orally harvested autogenous block grafts for vertical ridge augmentation in 12 patients. The study concluded that mandibular block autogenous bone grafts can maintain their vitality when used for vertical ridge augmentation and provide promising outcome in terms of gain in ridge height. ${ }^{(13)}$ This is in accordance with the present study where statistically significant gain in alveolar ridge height and width were noted at the 6 months follow-up.

In the donor site, sulcular incision approach can be used safely if there is no periodontal disease and no crowns are present in the anterior teeth, thickness of the attached gingiva and depth of the vestibule are adequate. Through this approach, it is easier to access the symphysis region up to the maximum extent as per the guideline and also for obtaining additional bone blocks and scrapings. ${ }^{(10)}$

Piezo-electric surgery was chosen as it provides microprecision, selective cutting ability, maximum visibility and excellent healing. Healing is enhanced by utilizing ultrasound to create micropits at the base of the defect to trigger cellular response of healing mechanisms. Furthermore, it decreases the invasiveness of conventional osseous surgery by hastening the procedure. It also encourages tissue healing by using the bone removed during the osteoplasty procedure to graft the osseous defects. ${ }^{(14)}$ So peizo-electric unit was utilized in the present study to procure the autogenous bone graft from the donor site and to perform decortication in the recipient site.

Recipient site preparation was carried out as it is crucial for predictable incorporation of block bone graft. In order to achieve this, decortication and perforation the marrow spaces is done as it quickens re-vascularization. This will lead to two to ten times faster healing than the physiological healing. ${ }^{(15)}$

The common post-operative complications that occur following graft harvesting from the symphyseal region is pain, donor site exposure, chin ptosis, hematoma and lingual space infection in severe cases. ${ }^{(10)}$ Apart from post-operative pain, swelling and hematoma formation in 3 patients, no other severe complications were encountered. The 3 patients who presented with the above mentioned discomforts did not require additional treatment as the symptoms subsided within the next three to four days.

\section{CONCLUSION}

The results of the present interventional study suggest that horizontal and vertical ridge augmentation of class III ridge defects can be successfully achieved with autogenous block bone graft from chin. The additional use of piezo-electric surgery, particulate bone graft materials and GTR membranes ensure the predictable outcome of the augmentation procedure. Dental implants can be appended six months after surgical placement of the grafting materials.

\section{Acknowledgement: Nil \\ Conflict of interest: Nil}

\section{REFERENCES}

1) Chavda S, Levin L. Human Studies of Vertical and Horizontal Alveolar Ridge Augmentation Comparing Different Types of Bone Graft Materials: A Systematic Review. Journal of Oral Implantology. 2018;44(1):74-84. Available from: https://dx.doi.org/10.1563/aaidjoi-d-17-00053.

2) 'ADdona $\mathrm{AD}$, Nowzari $\mathrm{H}$. Intramembranous autogenous osseous transplants in aesthetic treatment of alveolar atrophy. Periodontology 2000. 2001;27(1):148-161. Available from: https://dx.doi.org/10.1034/ j.1600-0757.2001.027001148.x.

3) Pikos MA. Alveolar ridge augmentation using mandibular block grafts: clinical update. Alpha Omegan. 2000;93:14-21.

4) Vercellotti T. Technological characteristics and clinical indications of piezoelectric bone surgery. Minerva stomatol. 2004;53:207-214.

5) Pavlíková G, Foltán R, Horká M, Hanzelka T, Borunská H, Šedý J. Piezosurgery in oral and maxillofacial surgery. International Journal of Oral and Maxillofacial Surgery. 2011;40(5):451-457. Available from: https://dx.doi.org/10.1016/j.ijom.2010.11.013.

6) Seibert JS. Reconstruction of deformed, partially edentulous ridges, using full thickness onlay grafts. Part I. Technique and wound healing. Compend Contin Educ Dent. 1983;4:437-453.

7) Pommer B, Tepper G, Gahleitner A, Zechner W, Watzek G. New safety margins for chin bone harvesting based on the course of the mandibular incisive canal in CT. Clinical Oral Implants Research. 2008;19(12):1312-1316. Available from: https://dx.doi.org/10.1111/j. 1600-0501.2008.01590.x

8) McAllister BS, Haghighat K. Bone Augmentation Techniques. Journal of Periodontology. 2007;78(3):377-396. Available from: https://dx.doi. org/10.1902/jop.2007.060048.

9) Chappuis V, Cavusoglu Y, Buser D, von Arx T. Lateral Ridge Augmentation Using Autogenous Block Grafts and Guided Bone Regeneration: A 10-Year Prospective Case Series Study. Clinical Implant Dentistry and Related Research. 2017;19(1):85-96. Available from: https://dx.doi.org/10.1111/cid.12438.

10) Pikos MA. Mandibular Block Autografts for Alveolar Ridge Augmentation. Atlas of the Oral and Maxillofacial Surgery Clinics. 2005;13(2):91-107. Available from: https://dx.doi.org/10.1016/j.cxom. 2005.05.003.

11) Misch CM. Comparison of intraoral donor sites for onlay grafting prior to implant placement. Int J Oral Maxillofac Implants. 1997;12:173-195.

12) Chiapasco M, Abati S, Romeo E, Vogel G. Clinical outcome of autogenous bone blocks or guided bone regeneration with ePTFE membranes for the reconstruction of narrow edentulous ridges. Clinical Oral Implants Research. 1999;10(4):278-288. Available from: https://dx.doi.org/10.1034/j.1600-0501.1999.100404.x.

13) Proussaefs P, Lozada J, Kleinman A, Rohrer MD, Mcmillan PJ. The use of titanium mesh in conjunction with autogenous bone graft and

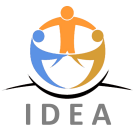


inorganic bovine bone mineral (Bio-Oss) for localized alveolar ridge augmentation: a human study. Int J Periodontics Restorative Dent. 2003;23:185-195.

14) Leclercq P, Zenati C, Dohan DM. Ultrasonic bone cut part 2: State-of-the-art specific clinical applications. J Oral Maxillofac Surg. 2008;661:183-188.
15) Rompen EH, Biewer R, Vanheusden A, Zahedi S, Nusgens B. The influence of cortical perforations and of space filling with peripheral blood on the kinetics of guided bone generation. A comparative histometric study in the rat. Clinical Oral Implants Research. 1999;10(2):85-94. Available from: https://dx.doi.org/10.1034/j.16000501.1999.100202.x. 